This is the peer reviewed version of the following article:

Kepic D., Sandoval S., Pino Á.P.D., György E., Cabana L., Ballesteros B., Tobias G.. Nanosecond Laser-Assisted Nitrogen Doping of Graphene Oxide Dispersions. ChemPhysChem, (2017). 18. : 935 - . 10.1002/cphc.201601256,

which has been published in final form at https: //dx.doi.org/10.1002/cphc.201601256. This article may be used for non-commercial purposes in accordance with Wiley Terms and Conditions for Use of Self-Archived Versions. 


\title{
Nanosecond laser-assisted nitrogen doping of graphene oxide dispersions
}

\author{
Dejan Kepić, ${ }^{\ddagger}[a]$, [b] Stefania Sandoval, ${ }^{\ddagger}[a]$ Ángel Pérez del Pino, ${ }^{[a]}$ Enikö György, ${ }^{[a], ~[c] ~ L a u r a ~ C a b a n a, ~}{ }^{[a]}$ \\ Belén Ballesteros, ${ }^{[d]}$ and Gerard Tobias ${ }^{*[a]}$
}

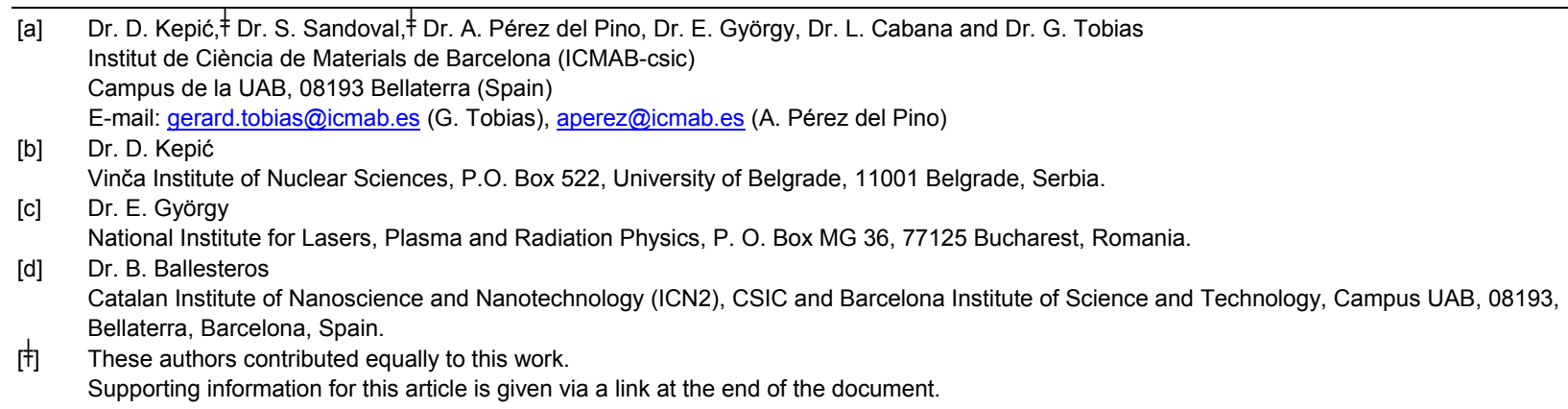

Abstract: N-doped reduced graphene oxide (RGO) has been prepared in bulk form by laser irradiation of graphene oxide (GO) dispersed in an aqueous solution of ammonia. A pulsed Nd:YAG laser with emission wavelengths in the infrared (IR) $1064 \mathrm{~nm}$, visible (VIS) $532 \mathrm{~nm}$, and ultraviolet (UV) $266 \mathrm{~nm}$ spectral regions was employed for the preparation of the N-doped RGO samples. Regardless of the laser energy employed, the resulting material presents a higher fraction of pyrrolic nitrogen compared to nitrogen atoms in pyridinic and graphitic coordination. Noticeably, whereas increasing the laser fluence of UV and VIS wavelengths results in an increase in the total amount of nitrogen, up to 4.9 at. \% (UV wavelength at $60 \mathrm{~mJ} \cdot \mathrm{cm}^{-2}$ fluence), the opposite trend is observed when the GO is irradiated in ammonia solution through IR processing. The proposed laser based methodology allows the bulk synthesis of $\mathrm{N}$-doped reduced graphene oxide in a simple, fast, and cost efficient manner.

\section{Introduction}

Graphene is a unique two-dimensional material that consists of a single layer of sp ${ }^{2}$-hybridized carbon atoms arranged in a honeycomb structure.$^{[1]} \mathrm{A}$ perfect graphene sheet is a zero-gap semiconductor since its conduction and valence bands meet at the Dirac points. However, the absence of a semiconducting bandgap hinders some of its potential applications. To overcome this problem, numerous methods have been proposed to introduce dopants into the graphene structure in order to tailor its electronic properties. ${ }^{[2]}$

Among the different doping strategies, the introduction of nitrogen heteroatoms is receiving an increased attention. The presence of nitrogen in the graphene lattice opens a narrow bandgap making the system an electron rich n-type semiconductor. The unique physical and chemical properties of $\mathrm{N}$-doped graphene find application in a wide range of areas, including biosensing, ${ }^{\left[{ }^{[3]}\right.}$ hydrogen storage ${ }^{[4]}$ and catalysis, ${ }^{[5]}$ to name some. The majority of doping strategies result in samples where the nitrogen heteroatoms are present in different coordination environments, namely pyridinic, pyrrolic and graphitic (or quaternary) ${ }^{[6]}$ Each type of nitrogen has a different contribution to the electronic structure of the material, ${ }^{[7]}$ and thus to the performance of $\mathrm{N}$-doped graphene for a given purpose. ${ }^{[8]}$ From the synthesis point of view this opens up new perspectives for the rational design of $\mathrm{N}$-doped graphene bearing a specific type of coordinated nitrogen to meet the requirements of a specific application. Therefore, to optimize the performance of $\mathrm{N}$-doped materials several strategies are being developed to foster the pyridinic, ${ }^{[9]}$ pyrrolic ${ }^{[8 b, 10]}$ or graphitic ${ }^{[11]}$ contribution. 
$\mathrm{N}$-doping can be performed either during the synthesis of graphene or via a post-synthesis modification of either individual graphene layers or the bulk material. For instance, individual layers and thin films of nitrogen doped graphene have been prepared via chemical vapour deposition, ${ }^{[4,12]}$ plasma enhanced chemical vapour deposition, ${ }^{[13]}$ low-energy $\mathrm{N}_{2}{ }^{+}$ion sputtering ${ }^{[14]}$ and laser irradiation. ${ }^{[15]}$ When it comes to bulk doping of graphene, the most commonly employed strategies involve hydrothermal treatment, ${ }^{[16]}$ microwave assisted synthesis, ${ }^{[17]}$ or thermal annealing in the presence of ammonia gas, ${ }^{[18]}$ being graphene oxide (GO) widely employed as starting material.

As a high power-density optical energy source, laser is capable of processing graphene and related materials in a controlled manner. Laser based methods are simple, rapid, and energy efficient compared to the aforementioned strategies for the preparation of $\mathrm{N}$-doped graphene. To date, most efforts with laser technologies have focused on the reduction of GO partly restoring the $\mathrm{sp}^{2}$ domains. GO has been laser treated in form of thin films mainly through infrared (IR) ${ }^{[19]}$ and ultraviolet (UV) wavelengths, ${ }^{[20]}$ and in liquid dispersions of the bulk material using UV ${ }^{[21]}$ and visible (VIS) radiations. ${ }^{[22]}$ Simultaneous reduction and doping of GO may occur during the irradiation of $\mathrm{GO}$ in the presence of reactive gases such as ammonia ${ }^{[23]}$ or chlorine. ${ }^{[24]}$ Laser irradiation of GO also finds application for dye removal from wastewaters ${ }^{[25]}$ and for the preparation of flexible organic photovoltaic cells. ${ }^{[26]}$ Despite that laser irradiation of GO in the presence of ammonia solution has been recently reported as an efficient reducing technique, ${ }^{[22]}$ the influence of the variation of the laser energy in the final composition of the sample (level of reduction) is not yet elucidated. Moreover, the possibility of using laserassisted technologies for doping bulk samples of graphene-based materials has not been investigated. Here we show that laser irradiation also arises as a competitive and efficient strategy for the introduction of nitrogen heteroatoms in the honeycomb structure. Several GO samples dispersed in aqueous ammonia have been irradiated using nanosecond pulsed lasers with different wavelengths $(\mathrm{UV}, \mathrm{VIS}, \mathrm{IR})$ and fluences $\left(20-900 \mathrm{~mJ} \cdot \mathrm{cm}^{-2}\right)$. The role that these laser irradiation parameters have on the level of doping, reduction as well as on the structure and morphology of the resulting $\mathrm{N}$-doped materials has been investigated.

\section{Results and Discussion}

The first evidence of laser-induced structural changes in the GO samples comes from visual inspection of the dispersions before and after irradiation. Whereas GO dispersed in ammonia presents a brownish colour, all the samples become darker after irradiation, regardless of the laser wavelength and fluence employed. The morphology of the different samples was investigated by means of transmission electron microscopy (TEM). Both single and few layered graphene were observed by TEM inspection of the sample. Figure 1 shows a TEM image and the corresponding selected area electron diffraction (SAED) pattern of the GO employed as starting material. The observed diffraction spots correspond to the (100) and (110) planes (d-spacing $=2.11 \AA$ and $1.23 \AA$, respectively) of the hexagonal lattice of the crystalline graphitic structure, ${ }^{[27]}$ which is mostly preserved upon oxidation. However, the spots appear blurred as a consequence of the corrugation of the GO sheet. ${ }^{[28]}$ According to the intensity of the inner and outer diffraction spots, the obtained pattern is in agreement with the presence of a single layer graphene sheet. ${ }^{[27]}$ 

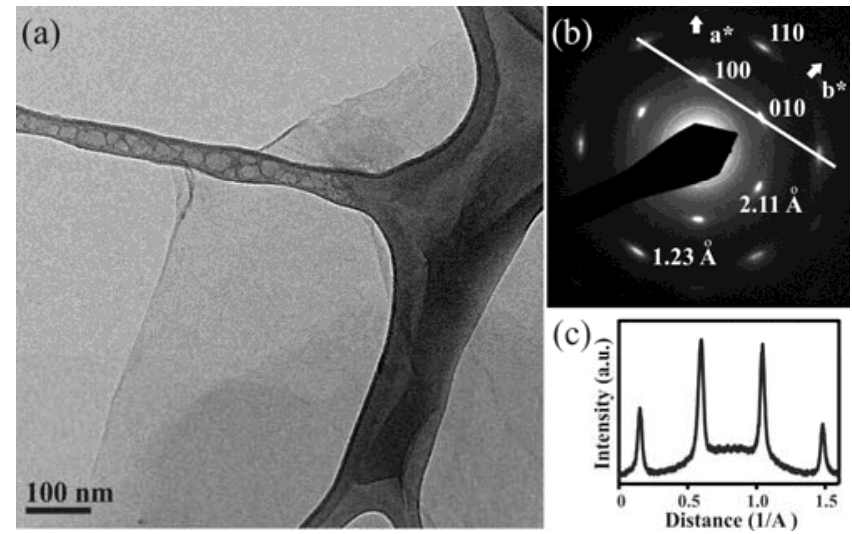

Figure 1. (a) TEM image and (b) SAED pattern of GO. (c) Corresponding intensity profile along the direction indicated with a white line in (b).

Representative TEM images of the samples after laser irradiation with different wavelengths and fluencies are presented in Figure 2, where slight variations in the appearance of the material are observed. In case of the UV irradiated samples, intensive wrinkling of the sheets is produced, resulting in a rag-shaped morphology (Figure 2(a)). The surface of the GO sheets irradiated using VIS laser shows the presence of spherically shaped areas with ca. $40 \mathrm{~nm}$ average diameters (Figure 2 (b), pointed by white arrows). The morphology of the GO sheets appears to be better preserved when the irradiation is performed with an IR wavelength at low fluences (Figure 2 (c)). However, similarly to the VIS irradiated samples, the highest applied fluence $\left(900 \mathrm{~mJ} \cdot \mathrm{cm}^{-2}\right)$ at this wavelength (IR) also shows the presence of the spherically shaped areas (Figure 2 (d)). HRTEM images were acquired to shed some light on the structure of these round shaped areas, which turned out to have anamorphous appearance lacking of crystallinity (Figure 2 (e,f) (dotted circle)). Thus, the laser-induced formation of other carbon allotropes such as graphitic particles or nanodiamonds, which synthesis has been previously reported upon laser irradiation of carbon-based materials, ${ }^{[29]}$ can be discarded.

Laser-induced creation of structural defects in graphene oxide based compounds has been previously observed. ${ }^{[20 \mathrm{~b}, 29 \mathrm{a}]}$ The radiation absorbed by GO sheets mainly leads to the development of extremely fast temperature variations of the material during the irradiation. The originated thermal energy would provoke chemical reactions of the functional groups present in the GO sheets, leading to the formation of structural defects in the graphene backbone. The accumulation of defects in combination with the laser pulse-induced thermal cycling would lead to the development of extremely high stress in the confined GO structure. Therefore, the origin of the observed wrinkled morphology could be attributed to the steady deformation of the GO irradiated sheets triggered by thermally created defects. Furthermore, more significant radiation-induced transformations will be provoked upon increasing the photon energy (lower wavelength) or laser fluence employed. Therefore, the spherical areas created by irradiation at high laser fluence might be due to fragments split from the graphene oxide. Apart from fast temperature variations, UV radiation can also prompt photochemical reactions since the photon energy $(4.7 \mathrm{eV})$ is larger than many of the activation energies of the chemical groups present in GO.[30] Thus, UV light lead to chemical and structural modifications of the GO structure, not achievable by means of VIS and IR radiation. This fact explains the higher quantity of structural defects observed in GO sheets irradiated with UV, as compared to VIS and IR irradiation (Figure 2). 


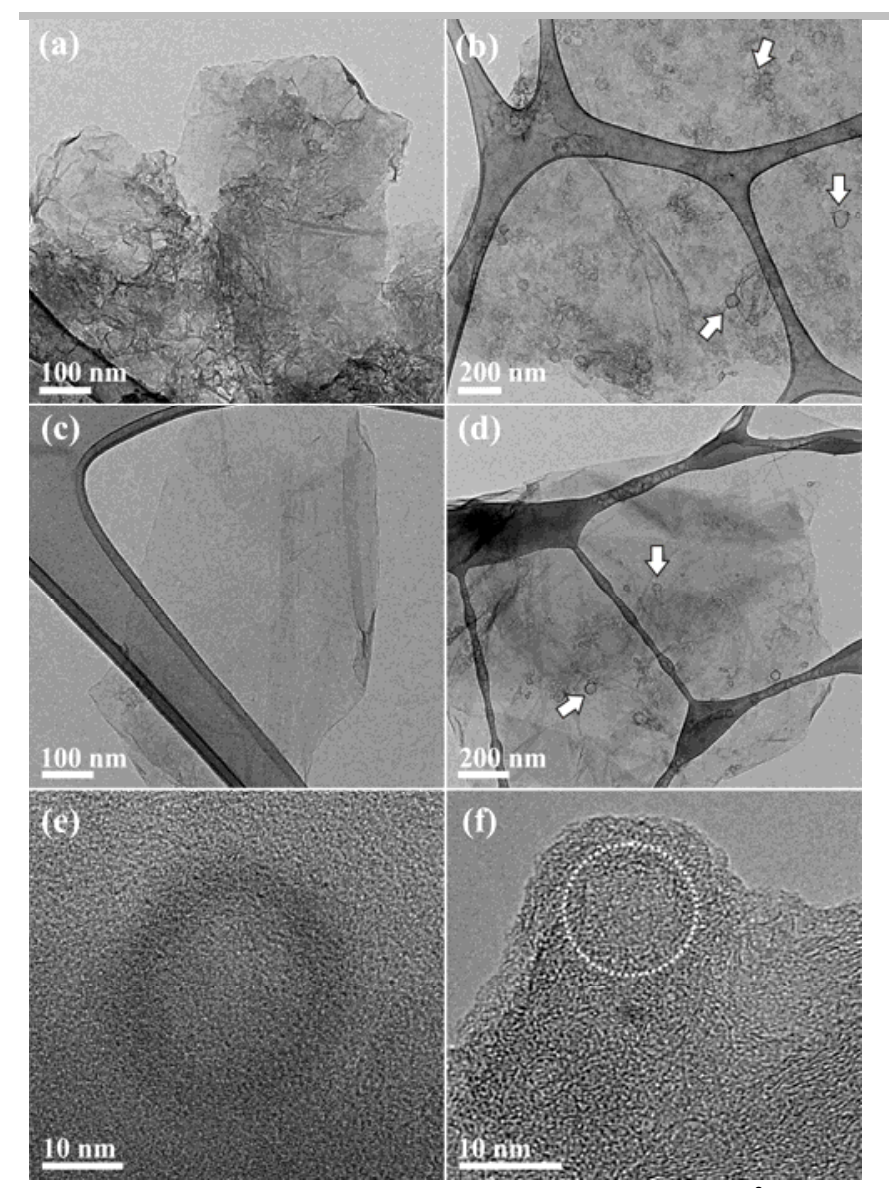

Figure 2.TEM images of samples irradiated with (a) UV- $60 \mathrm{~mJ} \cdot \mathrm{cm}^{-2}$, (b)VIS-300 $\mathrm{mJ} \cdot \mathrm{cm}^{-2}$, (c) IR $-300 \mathrm{~mJ} \cdot \mathrm{cm}^{-2}$ and (d) IR $-900 \mathrm{~mJ} \cdot \mathrm{cm}^{-2}$. (e, f) HRTEM images of samples irradiated with (e) VIS $-300 \mathrm{~mJ} \cdot \mathrm{cm}^{-2}$ and (f) IR $-900 \mathrm{~mJ} \cdot \mathrm{cm}^{-2}$.

The laser induced structural changes on the GO irradiated samples are also reflected in their Raman spectra (Figure S1, Supporting Information). For ease of comparison, the Raman spectrum of the reference material (non-irradiated $\mathrm{GO}$ in $\mathrm{NH}_{3}$ ) along with the UV, VIS, and IR laser treated samples with the same fluence of $60 \mathrm{~mJ} \cdot \mathrm{cm}^{-2}$ are presented in Figure 3. Two prominent peaks can be observed, which are characteristic of graphene oxide based materials. The peak centred at ca. $1350 \mathrm{~cm}^{-1}$ is related to the A1g symmetry breathing mode and arises from the presence of different types of structural defects in the aromatic rings (D-band), while the peak located at ca. $1590 \mathrm{~cm}^{-1}$, known as G-band, is related to well-ordered $\mathrm{sp}^{2}$-type bonds in the planar structure of graphite based materials. ${ }^{[31]}$ Three additional peaks located at ca. $1140 \mathrm{~cm}^{-1}, 2680 \mathrm{~cm}^{-1}$, and $2940 \mathrm{~cm}^{-1}$ are also observed that correspond to N-H in plane deformations, ${ }^{\text {[2] }}$ $2 D$ and $D+G$ bands, ${ }^{[33]}$ respectively. The intensity ratio of the $D$ and $G$ bands $\left(I_{D} / I_{G}\right)$ is commonly employed to estimate the disorder level of the $\mathrm{sp}^{2}$ graphene structure. ${ }^{[34]}$ All the irradiated samples show a slight increase in the $I_{D} / l_{G}$ ratio with respect to the reference material (Table S1Supporting Information). This might account for the creation of structural defects, in agreement with the TEM observations (Figure 2), and for the incorporation of $\mathrm{N}$ atoms into the RGO. ${ }^{[35]}$ Another consequence of the presence of structural defects and the $\mathrm{N}$ doping of RGO is the considerably small intensity of the $2 \mathrm{D}$ band. ${ }^{[36]}$ 


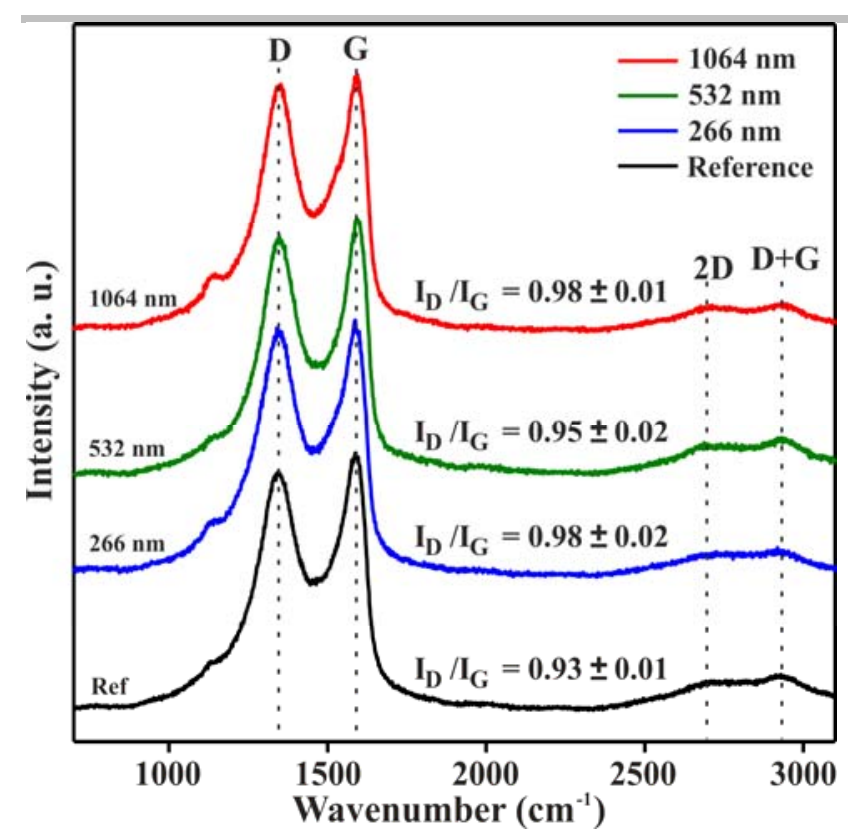

Figure 3. Raman spectra of the reference (non-irradiated $\mathrm{GO}$ in $\left.\mathrm{NH}_{3}\right)$ and irradiated samples with UV (266 nm), VIS (532 nm), and IR (1064 nm) wavelengths at 60 $\mathrm{mJ} \cdot \mathrm{cm}^{-2}$ laser fluence.

Next we assessed the degree of reduction of the samples by the laser treatment. Although XRD has been widely employed for this purpose, ${ }^{[37]}$ due to the small amount of sample obtained with the employed experimental set-up we used XPS. XPS analysis allows assessing both the degree of reduction and the amount of nitrogen incorporated into the structure through the quantification of the nitrogen, oxygen and carbon atomic contents. Furthermore, since different chemical environments are associated to specific binding energies, it is possible to discern the nitrogen-based functionalities introduced after the laser treatments. As expected, the general survey scans confirm the presence of carbon (C1s ca. $284 \mathrm{eV})$, oxygen (O1s ca. $530 \mathrm{eV})$ and nitrogen (N1s ca. $400 \mathrm{eV})$ for all the samples. The presence of nitrogen can actually be already detected in the reference sample, prepared by dispersing $\mathrm{GO}^{\text {in }} \mathrm{NH}_{3}$ at room temperature. Oxygen bearing functionalities attached to the graphitic network, namely carboxylic, hydroxyl or epoxy groups, can easily interact with ammonia containing solutions, leading to the formation of amino-functionalized GO. ${ }^{[38]}$ However, despite that an important amount of $\mathrm{N}$ is present in the reference sample (2.0 at. \%), there are no significant changes in both, $\mathrm{C}$ and $\mathrm{O}$ contents when compared to GO (Table 1), suggesting that the sample did not suffer any modifications in the conjugated network, i.e. no doping has taken place. Thus, the $\mathrm{N}$ atoms introduced in the reference material may correspond to $\mathrm{N}$-bearing aliphatic moieties, formed by a nucleophilic attack of $\mathrm{NH}_{3}$ onto the O-containing fractions. 
Table 1. Calculated atomic concentrations of $\mathrm{C}, \mathrm{O}$, and $\mathrm{N}$ content of samples irradiated by UV ( $266 \mathrm{~nm})$, VIS (532 nm), and IR (1064 nm) as determined by XPS.

\begin{tabular}{|c|c|c|c|c|}
\hline Sample & $\begin{array}{c}\text { Fluence } \\
\left(\mathrm{mJ} \cdot \mathrm{cm}^{-2}\right)\end{array}$ & C (at. \%) & $\mathrm{O}($ at. $\%)$ & $\mathrm{N}$ (at. \%) \\
\hline GO & -- & 66.6 & 32.8 & 0.6 \\
\hline $\begin{array}{c}\mathrm{GO}, \mathrm{NH}_{3} \\
\text { (Reference) }\end{array}$ & -- & 65.5 & 32.5 & 2.0 \\
\hline \multirow{2}{*}{$\begin{array}{c}\text { GO, } \mathrm{NH}_{3} \\
\text { UV } \\
(266 \mathrm{~nm})\end{array}$} & 20 & 76.2 & 19.3 & 4.5 \\
\hline & 60 & 75.9 & 19.2 & 4.9 \\
\hline \multirow{3}{*}{$\begin{array}{c}\mathrm{GO}, \mathrm{NH}_{3} \\
\text { VIS } \\
(532 \mathrm{~nm})\end{array}$} & 60 & 71.8 & 26.2 & 2.0 \\
\hline & 150 & 73.3 & 24.3 & 2.4 \\
\hline & 300 & 76.9 & 19.8 & 3.3 \\
\hline \multirow{4}{*}{$\begin{array}{c}\mathrm{GO}, \mathrm{NH}_{3} \\
\text { IR } \\
(1064 \mathrm{~nm})\end{array}$} & 60 & 70.7 & 26.3 & 3.0 \\
\hline & 150 & 71.6 & 25.8 & 2.6 \\
\hline & 300 & 70.5 & 27.0 & 2.5 \\
\hline & 900 & 71.8 & 25.9 & 2.3 \\
\hline
\end{tabular}

The high-resolution C1s XPS provides information about the distribution of the aliphatic groups attached to the conjugated lattice. In case of GO, the highest contribution corresponds to ketone groups (27.8 at. \%), followed by a 15 at. \% of carboxylic species and 9.2 at $\%$ of carbon linked to oxygen atoms by single bonds; Table $\mathrm{S} 2$, Supporting Information). After dispersing the material in the $\mathrm{NH}_{3}$ solution the appearance of a new peak, usually assigned to C-N bonds is observed. ${ }^{[6 \mathrm{~b}]}$ The $62.7 \%$ decrease in the concentration of the carboxylic groups suggests that $\mathrm{NH}_{3}$ mainly interact with these species to produce $\mathrm{N}$-derivatives. The contribution of the $\mathrm{C}=\mathrm{C}$ (284.8 eV) signal remains almost invariable, confirming that the conjugated lattice has been scarcely modified during the process (Figure S2 $(\mathrm{a}-\mathrm{b}))$.

As expected, all the laser treated samples undergo a partial reduction. ${ }^{[39]}$ This is reflected by an increase in the relative amount of carbon content, from 65.5 at. \% in the reference material to 70.7-76.9 at. \%, depending on the photon energy and fluence of the employed laser. Figure 4 (a) shows the variation of both $\mathrm{C}$ (squares) and $\mathrm{O}$ (circles) concentration after irradiation of the $\mathrm{GO}-\mathrm{NH}_{3}$ dispersions with UV, VIS and IR energies. A higher degree of reduction is obtained by employing a higher photon energy, which can be appreciated when comparing samples treated at the same fluence $\left(60 \mathrm{~mJ} \cdot \mathrm{cm}^{-2}\right)$ under different laser photon energies $(75.9$ at. $\%$, 71.8 at. $\%$ and 70.7 at. \% C for UV, VIS and IR lasers, respectively). XPS spectra of the N-doped RGO irradiated with UV and VIS light are presented in Figure S2 (c-d). Following the same trend, the highest level of nitrogen is introduced when employing the highest photon energy (UV), achieving contents of 4.9 at. \% N. VIS and IR irradiations lead to much lower nitrogen content, in the range of 2.0 3.3 at. \%. The amount of nitrogen that can be introduced with the present approach is similar to the values commonly achieved by other reported protocols, including chemical vapour deposition growth of N-doped graphene and bulk synthesis of N-doped graphenebased materials via thermal, solvothermal and electrochemical methods. [6b, 40] 

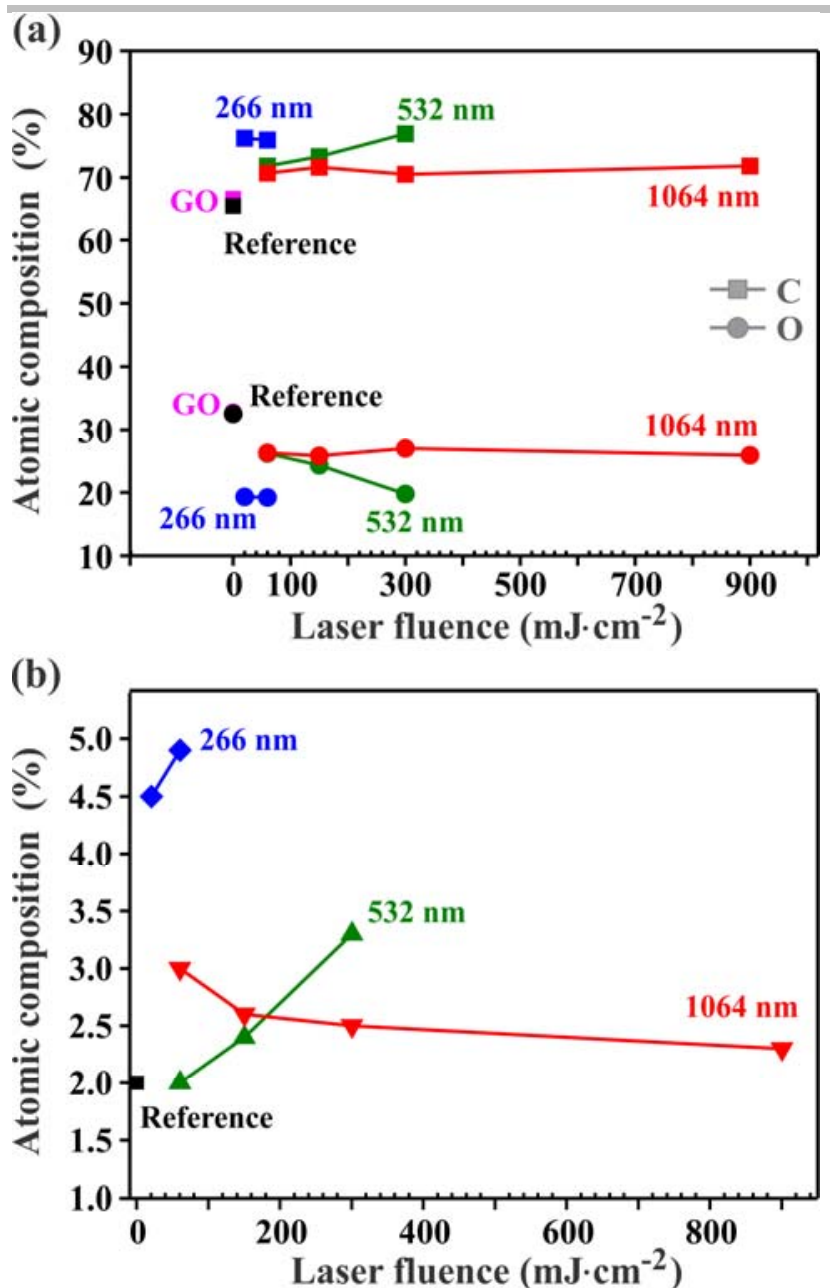

Figure 4. (a) Dependence of carbon and oxygen atomic concentration on the laser fluence. Samples were irradiated with UV (266 $\mathrm{nm}$ ), VIS (532 $\mathrm{nm}$ ) and IR (1064 nm). (b) Nitrogen content present in the samples after laser treatments.

The role of the laser fluence on the amount of nitrogen present in the samples deserves some attention. Whereas an increase in the laser fluence, i. e. number of photons, results in an increase in the nitrogen content for both the UV and VIS wavelengths, the opposite trend is observed under IR irradiation, with a decrease in the nitrogen content from 3.0 at. $\%\left(60 \mathrm{~mJ} \cdot \mathrm{cm}^{-2}\right)$ to 2.3 at. $\%\left(900 \mathrm{~mJ} \cdot \mathrm{cm}^{-2}\right)$. To better appreciate the influence of the different experimental parameters, Figure 4(b) summarizes the nitrogen content with respect to the laser wavelengths and fluencies employed.

Next, the type of nitrogen present in the irradiated samples was determined by deconvolution of the high resolution XPS spectra over the N1s area. The N1s signal was deconvoluted to three peaks centred at $398.6 \mathrm{eV}, 399.8 \mathrm{eV}$ and $401.8 \mathrm{eV}$, which have been respectively assigned to pyridinic, pyrrolic and graphitic nitrogen in $\mathrm{N}$-doped materials. ${ }^{[41]}$ The position of the two peaks at lower binding energies overlaps with that of aliphatic amine/amide groups ${ }^{[18 \mathrm{~b}, 20 \mathrm{~b}]}$ and therefore, special care is needed when using XPS to assess the degree of doping. In the present study, the presence of graphitic $\mathrm{N}$ suggests that structural $\mathrm{N}$ is introduced into the sample by laser/ $\mathrm{NH}_{3}$ treatments (Figures S2-S4, Supporting Information). Additional evidence of doping arises from the observed agglomeration of the material upon irradiation, since the presence of $\mathrm{N}$-bearing aliphatic functionalities would result in highly dispersible samples. ${ }^{[42]}$ The doping process is produced via the interaction of the oxygen based moieties on the GO surface and the nitrogen source. We analysed the variation on the concentration of these species in the samples to confirm the presence of structural $\mathrm{N}$ (introduced in the conjugated lattice) upon irradiation (Table S2, Supporting Information). Initially, GO is dispersed in aqueous ammonia (reference sample) prior to its irradiation by laser to afford the N-doped RGO. Therefore, GO undergoes aliphatic functionalization of its surface 
and thus the reference material still contains a significant contribution from $\mathrm{C}=\mathrm{O}(27.2 \%$, from deconvolution of XPS C1s). A dramatic decrease of $\mathrm{C}=\mathrm{O}$ in the $\mathrm{C} 1 \mathrm{~s}$ region is observed by subsequent treatment under UV, VIS and IR lasers (down to $3.2 \%-7.7 \%$ ) which is accompanied by an increase in the contribution of the $\mathrm{C}=\mathrm{C}$ signal. This implies that in the present study, the aliphatic O-based groups in $\mathrm{GO}$ are initially functionalized forming $\mathrm{N}$-containing aliphatic species when the samples are dispersed in $\mathrm{NH}_{3}$. The laser irradiation induces rearrangements and the subsequent incorporation of the $\mathrm{N}$ atoms in the conjugated system. Nevertheless, despite having experimental evidence of doping, the presence of $\mathrm{N}$-bearing aliphatic functionalities cannot be completely ruled out, since both doping and $\mathrm{N}$-aliphatic groups might coexist. ${ }^{[42]}$

It should be noted that all the irradiated samples have the highest contribution from pyrrolic nitrogen, reaching up to $71.4 \%$ of the total $\mathrm{N}$ content for the UV treated sample at $60 \mathrm{~mJ} \cdot \mathrm{cm}^{-2}$ (Table 2). This behaviour is in agreement with the studies of Guo et al. on laser irradiation of $\mathrm{GO}$ films in the presence of $\mathrm{NH}_{3}$ gas, where the formation of pyrrolic nitrogen is also favoured. ${ }^{[23]}$ Graphene enriched with pyrrolic nitrogen has shown good performance, for instance, as electrical double layer supercapacitor ${ }^{[43]}$ and metal-free electrocatalyst. ${ }^{[44]}$

Table 2. Calculated atomic concentration of different types of $\mathrm{N}$ based on high-resolution XPS spectra

\begin{tabular}{|c|c|c|c|c|c|}
\hline Sample & $\begin{array}{l}\text { Fluence } \\
\left(\mathrm{mJ} \cdot \mathrm{cm}^{-2}\right)\end{array}$ & Content & Pyridinic N & Pyrrolic N & Graphitic N \\
\hline \multirow{4}{*}{$\begin{array}{c}\text { GO, } \mathrm{NH}_{3} \\
\text { UV (266 nm) }\end{array}$} & \multirow{2}{*}{20} & at. $\%$ & 1.0 & 3.1 & 0.4 \\
\hline & & $100 \% \mathrm{~N}$ & $22.2 \%$ & $68.9 \%$ & $8.9 \%$ \\
\hline & \multirow{2}{*}{60} & at. $\%$ & 1.0 & 3.5 & 0.4 \\
\hline & & $100 \% \mathrm{~N}$ & $20.4 \%$ & $71.4 \%$ & $8.2 \%$ \\
\hline \multirow{6}{*}{$\begin{array}{c}\mathrm{GO}, \mathrm{NH}_{3}, \\
\text { VIS (532 nm) }\end{array}$} & \multirow{2}{*}{60} & at. $\%$ & 0.6 & 1.2 & 0.2 \\
\hline & & $100 \% \mathrm{~N}$ & $30 \%$ & $60 \%$ & $10 \%$ \\
\hline & \multirow{2}{*}{150} & at. $\%$ & 0.5 & 1.4 & 0.5 \\
\hline & & $100 \% \mathrm{~N}$ & $20.8 \%$ & $58.4 \%$ & $20.8 \%$ \\
\hline & \multirow{2}{*}{300} & at. $\%$ & 0.7 & 2.0 & 0.6 \\
\hline & & $100 \% \mathrm{~N}$ & $21.2 \%$ & $60.6 \%$ & $18.2 \%$ \\
\hline \multirow{8}{*}{$\begin{array}{c}\mathrm{GO}, \mathrm{NH}_{3} \\
\operatorname{IR}(1064 \mathrm{~nm})\end{array}$} & \multirow{2}{*}{60} & at. $\%$ & 0.5 & 1.8 & 0.7 \\
\hline & & $100 \% \mathrm{~N}$ & $16.7 \%$ & $60 \%$ & $23.3 \%$ \\
\hline & \multirow{2}{*}{150} & at. $\%$ & 0.5 & 1.7 & 0.4 \\
\hline & & $100 \% \mathrm{~N}$ & $19.2 \%$ & $65.4 \%$ & $15.4 \%$ \\
\hline & \multirow{2}{*}{300} & at. $\%$ & 0.5 & 1.7 & 0.3 \\
\hline & & $100 \% \mathrm{~N}$ & $20 \%$ & $68 \%$ & $12 \%$ \\
\hline & \multirow{2}{*}{900} & at. $\%$ & 0.6 & 1.5 & 0.2 \\
\hline & & $100 \% \mathrm{~N}$ & $26.1 \%$ & $65.2 \%$ & $8.7 \%$ \\
\hline
\end{tabular}

The different chemical behaviour (reduction and N-doping) of GO submitted to IR, VIS, and UV laser pulses (Table 2) could be essentially related to the activation energy of different chemical processes. While thermal and photo-induced migration of species for the initiation of chemical reactions would require low energy, attainable by IR photons (1.2 eV photon energy), different photo-activated chemical reaction pathways would appear by the action of larger energy VIS $(2.3 \mathrm{eV})$ and UV (4.7 eV) photons. ${ }^{[30]}$

To complete the study, both the reference material and the irradiated samples were next investigated by UV-Vis spectroscopy, since this technique allows monitoring changes in the electronic transitions of GO caused by N-doping. UV-Vis spectra of samples processed with UV, VIS, and IR radiation at $60 \mathrm{~mJ} \cdot \mathrm{cm}^{-2}$ laser fluence are presented in Figure 5 . The spectrum of the reference sample has two characteristic signals also present in graphene oxide, corresponding to the $\pi-\Pi^{*}$ (ca. $230 \mathrm{~nm}$ ) and n- $\pi^{*}$ (ca. $300 \mathrm{~nm}$ ) electron transitions, generally associated to the $\mathrm{C}=\mathrm{C}$ and $\mathrm{C}=\mathrm{O}$ groups present in the conjugated lattice and the aliphatic oxygen-functionalities respectively. ${ }^{[45]}$ In all the irradiated samples a red shift of the $\pi-\pi^{*}$ peak can be observed. This shift can be directly related to the level of reduction of the material and the nitrogen content. ${ }^{[42]}$ The change of peak position could thus be ascribed to partial restoration of electronic conjugation of GO generally produced by the elimination of the oxygen based functionalities (defects) present into the lattice. ${ }^{[21 a, 46]}$ On the other hand, the higher the nitrogen content, the higher the shift to the red region is observed. ${ }^{[42]}$ The nitrogen 
contribution to the red shift can be better appreciated comparing the VIS and IR samples which have the same amount of oxygen (ca. $26.2 \%$ ) but different amounts of nitrogen (2.0 and 3.0 at. \%, respectively). Following the discussion the IR sample presents a higher red shift $(236 \mathrm{~nm})$ than the VIS treated sample $(233 \mathrm{~nm})$ with respect to the reference material $(230 \mathrm{~nm})$. Additionally, the highest decrease in the $\mathrm{n}-\pi^{*}$ transition band is observed for the sample containing the highest amount of $\mathrm{N}$-based functionalities confirming the relation between the elimination of oxygen bearing functionalities from the GO lattice and the level of doping. ${ }^{[47]}$

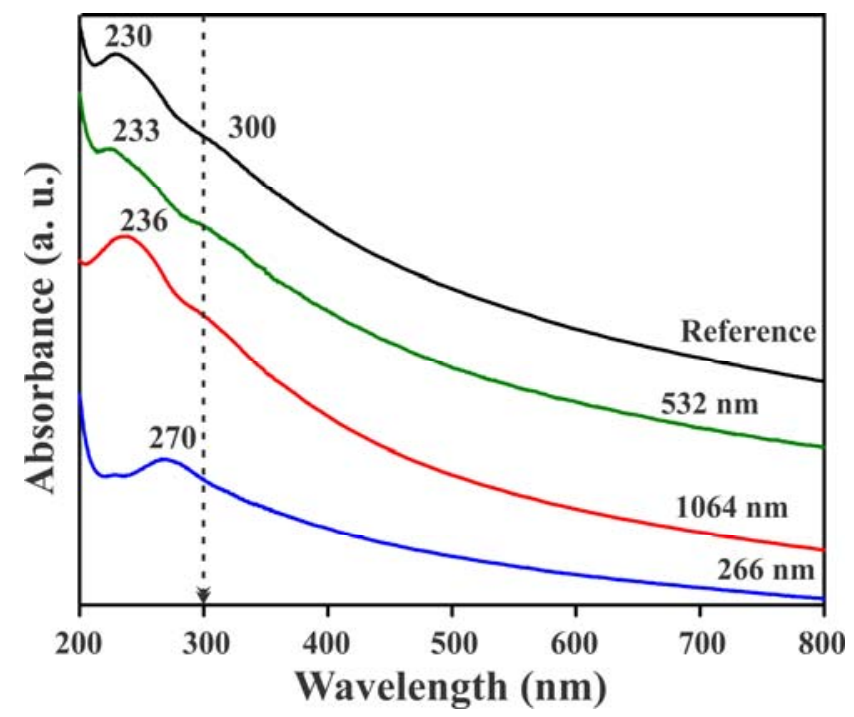

Figure 5.UV-Vis spectra of reference and samples irradiated with UV, VIS, and IR laser pulses and $60 \mathrm{~mJ} \cdot \mathrm{cm}^{-2}$ fluence.

\section{Conclusions}

Laser irradiation has been employed for the preparation of $\mathrm{N}$-doped RGO, further expanding the protocols available for the bulk synthesis of $\mathrm{N}$-doped graphene-related materials. The laser-assisted doping goes via the irradiation of $\mathrm{GO}$ in an aqueous solution of ammonia. The role of both the laser energy (UV, visible, and IR) and fluence on the resulting material has been investigated by means of TEM, XPS, Raman and UV-Vis spectroscopies. Whereas UV (266 nm) and visible $(532 \mathrm{~nm})$ laser irradiations yield to higher nitrogen doping with the increase of the laser fluence, the opposite trend is observed by the IR (1064 nm) processing. The obtained results point out that mainly thermal mechanisms, induced by IR radiation, provoke different reduction and N-doping chemical paths than those influenced by additional photochemical ones, induced by visible and UV radiation. Regardless of the laser energy and fluence employed, all the laser treated samples present a larger amount of pyrrolic nitrogen than pyridinic and graphitic fractions, reaching relative $\mathrm{N}$ pyrrolic contents of $71.4 \%$. The highest level of $\mathrm{N}$-doping $\left(4.9\right.$ at. \% N) was achieved with UV laser radiation at a fluence of $60 \mathrm{~mJ} \cdot \mathrm{cm}^{-}$

2. This level of doping is within the range achieved by most doping strategies. The most important advantages of the proposed method for the synthesis of $\mathrm{N}$-doped reduced graphene oxide lie in its simplicity, rapidness (nanoseconds), and energy efficiency.

\section{Experimental Section}

Synthesis of GO

$\mathrm{GO}$ was prepared via a modified Hummers' method. Concentrated $\mathrm{H}_{2} \mathrm{SO}_{4}(46 \mathrm{~mL})$ and $\mathrm{NaNO}_{3}(1 \mathrm{~g})$ were mixed with $2 \mathrm{~g}$ of graphite powder $(20 \mu \mathrm{m}$, Sigma-Aldrich), and cooled down to $0{ }^{\circ} \mathrm{C}$. After $30 \mathrm{~min}, \mathrm{KMnO}_{4}$ was added slowly $(6 \mathrm{~g})$ keeping the temperature of the solution below $20^{\circ} \mathrm{C}$. The reaction mixture was warmed to $35^{\circ} \mathrm{C}$ and stirred for $30 \mathrm{~min}$. Afterwards, $100 \mathrm{~mL}$ of distilled water were added and the temperature was raised to $98^{\circ} \mathrm{C}$ and held 
for $2 \mathrm{~h}$. Then, additional water $(400 \mathrm{~mL})$ and a $30 \% \mathrm{H}_{2} \mathrm{O}_{2}$ solution $(2 \mathrm{~mL})$ were added slowly. The content was cooled down, purified several times by washing with distilled water followed by centrifugation until the $\mathrm{pH}$ of the solution was neutral. Finally, the sample was collected as a solid powder and dried at $60^{\circ} \mathrm{C}$.

\section{Laser irradiation}

GO was dispersed in a 30 wt. \% ammonia aqueous solution using an ultrasound bath to obtain a GO concentration of $1 \mathrm{mg} / \mathrm{mL}$. A volume of $3 \mathrm{~mL}$ of GO/ammonia dispersion was then placed in quartz cuvettes with stirrers. Pulsed laser treatments were done by means of a Nd:YAG laser system (Brilliant B model from Quantel) which delivers pulses in the 5-6 ns range. Samples were irradiated employing different laser wavelengths, namely 266 nm (UV), $532 \mathrm{~nm}(\mathrm{VIS})$, and $1064 \mathrm{~nm}$ (IR), being the respective photon energies $4.7 \mathrm{eV}, 2.3 \mathrm{eV}$ and $1.2 \mathrm{eV}$. 2000 laser pulses were accumulated for each sample. The solutions were continuously stirred with a rotation frequency of $500 \mathrm{rpm}$. The irradiation was conducted by applying different laser fluences for each wavelength (20 and $60 \mathrm{~mJ} \cdot \mathrm{cm}^{-2}$ for $266 \mathrm{~nm} ; 60,150$, and $300 \mathrm{~mJ} \cdot \mathrm{cm}^{-2}$ for $532 \mathrm{~nm}$; and $60,150,300$, and $900 \mathrm{~mJ} \cdot \mathrm{cm}^{-2}$ for $1064 \mathrm{~nm}$ ). After irradiation, samples were filtered using polycarbonate membranes $(0.2 \mu \mathrm{m}$ pore size), thoroughly washed with distilled water to remove residual ammonia and dried at $60{ }^{\circ} \mathrm{C}$. To determine any potential interaction/reaction between the aliphatic functional groups present in the $\mathrm{GO}$ sheets and $\mathrm{NH}$, a reference, nonirradiated sample was also prepared by dispersing GO in the ammonia solution following the same protocol employed for the solutions submitted to laser irradiation.

\section{Characterization}

The morphology of the samples and selected area electron diffraction (SAED) patterns were recorded by transmission electron microscopy employing a TEM JEOL Jem 1210 electron microscope operating at $120 \mathrm{kV}$. High resolution images were acquired at $200 \mathrm{kV}$ on an FEI Tecnai G2 F20 microscope. Samples were prepared sonicating and dispersing in hexane. Afterwards, they were placed dropwise onto a lacey carbon support grid. Raman spectra were obtained using a Horiba Jobin Yvon instrument, operating at $532 \mathrm{~nm}$ wavelength and using a 100× objective. The acquisition time was set to $30 \mathrm{~s}$ and laser power to $0.5 \mathrm{~mW}$. UV-Vis spectra were recorded on a Cary 5 UV-Vis-NIR spectrophotometer in the range of $200-800 \mathrm{~nm}$. Samples were prepared by sonication of the material in a 1:1 ethanol/water mixture and analyses were carried out in absorbance mode using $1 \mathrm{~cm}$ standard quartz cuvettes. X-ray photoelectron spectroscopy (XPS) measurements were recorded in a Kratos AXIS ultra DLD spectrometer using a monochromatic Al Ka. All samples were introduced in the preparation chamber as received and on the same substrate $(\mathrm{Cu})$ to maintain the analysis conditions invariable.

\section{Acknowledgements}

The research leading to these results has received funding from the Spanish Ministry of Economy and Competitiveness (MINECO, Spain) through the grants MAT2014-53500-R and ENE2014-56109-C3-3-R. ICMAB and ICN2 acknowledge financial support from the Spanish Ministry of Economy and Competitiveness, through the "Severo Ochoa" Programme for Centres of Excellence in R\&D (SEV2015-0496 and SEV-2013-0295). D.K. acknowledges the financial support of the Ministry of Education, Science and Technological Development of Republic of Serbia for postdoctoral research. S.S. acknowledges a contract though PIE 201660E013. The XPS data was acquired at the Laboratorio de Microscopías Avanzadas (LMA) - Instituto de Nanociencia de Aragón (INA).

Keywords: graphene $\bullet$ laser $・ \mathrm{~N}$-doped $\bullet$ ammonia $\bullet$ RGO

[1] a) A. H. Castro Neto, F. Guinea, N. M. R. Peres, K. S. Novoselov and A. K. Geim, Reviews of Modern Physics 2009, 81, 109-162; b) A. A. Balandin, Nature Materials 2011, 10, 569-581.

[2] a) E. Bekyarova, M. E. Itkis, P. Ramesh, C. Berger, M. Sprinkle, W. A. de Heer and R. C. Haddon, Journal of the American Chemical Society 2009, 131, 1336$1337 ;$ b) D. C. Elias, R. R. Nair, T. M. G. Mohiuddin, S. V. Morozov, P. Blake, M. P. Halsall, A. C. Ferrari, D. W. Boukhvalov, M. I. Katsnelson, A. K. Geim and K. S. Novoselov, Science 2009, 323, 610-613;c) S. Yu, W. Zheng, C. Wang and Q. Jiang, ACS Nano 2010, 4, 7619-7629.

[3] Y. Wang, Y. Shao, D. W. Matson, J. Li and Y. Lin, ACS Nano 2010, 4, 1790-1798.

[4] X.-K. Kong, Q.-W. Chen and Z.-Y. Lun, ChemPhysChem 2014, 15, 344-350.

[5] M. D. Esrafili, R. Mohammad-Valipour, S. M. Mousavi-Khoshdel and P. Nematollahi, ChemPhysChem 2015, 16, 3719-3727. 
[6] a) Functionalization of Graphene, Wiley-VCH, Somerset, DE, 2014; b) H. Wang, T. Maiyalagan and X. Wang, ACS Catalysis 2012, 2, 781-794.

[7] K. Akada, T.-o. Terasawa, G. Imamura, S. Obata and K. Saiki, Applied Physics Letters 2014, 104, 131602.

[8] a) X.-k. Kong and Q.-w. Chen, Physical Chemistry Chemical Physics 2013, 15, 12982-12987;b) T. Katoh, G. Imamura, S. Obata and K. Saiki, RSC Advances 2016, 6, 13392-13398;c) D. Guo, R. Shibuya, C. Akiba, S. Saji, T. Kondo and J. Nakamura, Science 2016, 351, 361-365;d) C. Zhan, Y. Zhang, P. T. Cummings and D.-e. Jiang, Physical Chemistry Chemical Physics 2016, 18, 4668-4674.

[9] a) S. Jiaguang, W. Lan, S. Ranran and Y. Shubin, Nanotechnology 2016, 27, 055404;b) M. S. Lee, H.-J. Choi, J.-B. Baek and D. W. Chang, Applied Energy;C) A. A. Ensafi, M. Jafari-Asl and B. Rezaei, Electrochimica Acta 2016, 194, 95-103; d) E. Rangel, L. F. Magana and L. E. Sansores, ChemPhysChem 2014, 15, 4042-4048.

[10] a) J. Li, X. Li, P. Zhao, D. Y. Lei, W. Li, J. Bai, Z. Ren and X. Xu, Carbon 2015, 84, 460-468;b) J. Li, Z. Ren, Y. Zhou, X. Wu, X. Xu, M. Qi, W. Li, J. Bai and L. Wang, Carbon 2013, 62, 330-336.

[11] a) T. N. Huan, T. Van Khai, Y. Kang, K. B. Shim and H. Chung, Journal of Materials Chemistry 2012, 22, 14756-14762;b) F. Yang, C. Chi, C. Wang, Y. Wang and Y. Li, Green Chemistry 2016, 18, 4254-4262.

[12] L. Qu, Y. Liu, J. B. Baek and L. Dai, ACS Nano 2010, 4, 1321-1326.

[13] H. M. Jeong, J. W. Lee, W. H. Shin, Y. J. Choi, H. J. Shin, J. K. Kang and J. W. Choi, Nano Letters 2011, 11, 2472-2477.

[14] a) L. Zhang, Y. Ye, D. Cheng, W. Zhang, H. Pan and J. Zhu, Carbon 2013, 62, 365-373;b) W. Zhao, O. Höfert, K. Gotterbarm, J. F. Zhu, C. Papp and H. P. Steinrück, Journal of Physical Chemistry C 2012, 116, 5062-5066.

[15] I. Choi, H. Y. Jeong, D. Y. Jung, M. Byun, C.-G. Choi, B. H. Hong, S.-Y. Choi and K. J. Lee, ACS Nano 2014, 8, 7671-7677.

[16] a) B. Jiang, C. Tian, L. Wang, L. Sun, C. Chen, X. Nong, Y. Qiao and H. Fu, Applied Surface Science 2012, 258, 3438-3443;b) J. Yang, M. R. Jo, M. Kang, Y. S. Huh, H. Jung and Y. M. Kang, Carbon 2014, 73, 106-113.

[17] K. Gopalakrishnan, A. Govindaraj and C. N. R. Rao, Journal of Materials Chemistry A 2013, 1, 7563-7565.

[18] a) S. Sandoval, N. Kumar, A. Sundaresan, C. N. R. Rao, A. Fuertes and G. Tobias, Chemistry - A European Journal 2014, 20, 11999-12003;b) X. Li, H. Wang, J. T. Robinson, H. Sanchez, G. Diankov and H. Dai, Journal of the American Chemical Society 2009, 131, 15939-15944;C) H. Wang, C. Zhang, Z. Liu, L. Wang, P. Han, H. Xu, K. Zhang, S. Dong, J. Yao and G. Cui, Journal of Materials Chemistry 2011, 21, 5430-5434.

[19] a) W. Gao, N. Singh, L. Song, Z. Liu, A. L. M. Reddy, L. Ci, R. Vajtai, Q. Zhang, B. Wei and P. M. Ajayan, Nature Nanotechnology 2011, 6, 496-500;b) M. F. El-Kady, V. Strong, S. Dubin and R. B. Kaner, Science 2012, 335, 1326-1330.

[20] a) E. György, A. Pérez del Pino, C. Logofatu, A. Duta and L. Isac, Journal of Applied Physics 2014, 116, 024906;b) A. Pérez del Pino, E. György, C. Logofatu, J. Puigmartí-Luis and W. Gao, Carbon 2015, 93, 373-383.

[21] a) L. Huang, Y. Liu, L. C. Ji, Y. Q. Xie, T. Wang and W. Z. Shi, Carbon 2011, 49, 2431-2436;b) Y. Matsumoto, M. Koinuma, S. Ida, S. Hayami, T. Taniguchi, K. Hatakeyama, H. Tateishi, Y. Watanabe and S. Amano, Journal of Physical Chemistry C 2011, 115, 19280-19286.

[22] E. E. Ghadim, N. Rashidi, S. Kimiagar, O. Akhavan, F. Manouchehri and E. Ghaderi, Applied Surface Science 2014, 301, 183-188.

[23] L. Guo, Y. L. Zhang, D. D. Han, H. B. Jiang, D. Wang, X. B. Li, H. Xia, J. Feng, Q. D. Chen and H. B. Sun, Advanced Optical Materials 2014, 2, 120-125.

[24] K. Savva, Y. H. Lin, C. Petridis, E. Kymakis, T. D. Anthopoulos and E. Stratakis, Journal of Materials Chemistry C 2014, 2, $5931-5937$.

[25] P. Russo, L. D'Urso, A. Hu, N. Zhou and G. Compagnini, Applied Surface Science 2015, 348, 85-91.

[26] E. Kymakis, K. Savva, M. M. Stylianakis, C. Fotakis and E. Stratakis, Advanced Functional Materials 2013, 23, 2742-2749.

[27] N. R. Wilson, P. A. Pandey, R. Beanland, R. J. Young, I. A. Kinloch, L. Gong, Z. Liu, K. Suenaga, J. P. Rourke, S. J. York and J. Sloan, ACS Nano 2009, 3, 2547-2556.

[28] J. C. Meyer, A. K. Geim, M. I. Katsnelson, K. S. Novoselov, T. J. Booth and S. Roth, Nature 2007, 446, 60-63.

[29] a) Á. Pérez del Pino, E. György, L. Cabana, B. Ballesteros and G. Tobias, Journal of Applied Physics 2014, 115, 093501;b) R.-F. Xiao and Z.-F. Li, Applied Surface Science 1998, 127-129, 826-830.

[30] D. A. Sokolov, Y. V. Morozov, M. P. McDonald, F. Vietmeyer, J. H. Hodak and M. Kuno, Nano Letters 2014, 14, 3172-3179.

[31] A. C. Ferrari, Solid State Communications 2007, 143, 47-57.

[32] M. L. Giuseppe Perna, Crescenzio Gallo, Giuseppe Quartucci, Vito Capozzi, The Open Surface Science Journal 2013, 5, 1-8.

[33] a) L. M. Malard, M. A. Pimenta, G. Dresselhaus and M. S. Dresselhaus, Physics Reports 2009, 473, 51-87;b) J. Hong, M. K. Park, E. J. Lee, D. Lee, D. S. Hwang and S. Ryu, Sci. Rep. 2013, 3.

[34] a) D. Pan, S. Wang, B. Zhao, M. Wu, H. Zhang, Y. Wang and Z. Jiao, Chemistry of Materials 2009, 21, 3136-3142;b) K. N. Kudin, B. Ozbas, H. C. Schniepp, R. K. Prud'homme, I. A. Aksay and R. Car, Nano Letters 2008, 8, 36-41.

[35] a) Z. M. Markovic, B. Z. Ristic, K. M. Arsikin, D. G. Klisic, L. M. Harhaji-Trajkovic, B. M. Todorovic-Markovic, D. P. Kepic, T. K. Kravic-Stevovic, S. P. Jovanovic M. M. Milenkovic, D. D. Milivojevic, V. Z. Bumbasirevic, M. D. Dramicanin and V. S. Trajkovic, Biomaterials 2012, 33, 7084-7092;b) M. Mowry, D. Palaniuk, C. C. Luhrs and S. Osswald, RSC Advances 2013, 3, 21763-21775.

[36] a) Z. Zafar, Z.H. Ni, X. Wu, Z.X. Shi, H.Y. Nan, J. Bai, L.T. Sun, Carbon 2013, 61, 57-62; b) C. Zhang, L. Fu, N. Liu, M. Liu, Y. Wang, Z. Liu, Advanced Materials 2011, 23, 1020-1024;c) P. Venezuela, M. Lazzeri, F. Mauri, Physical Review B 2011, 84, 035433.

[37] A. Alazmi, O. El Tall, S. Rasul, M.N. Hedhili, S.P. Patole, P.M.F.J. Costa, Nanoscale 2016, 8, 17782-17787

[38] L. Yang, J. Hu, W. Wu, J. Tang, K. Ding and J. Li, Chemical Engineering Journal 2016, 306, 77-85.

[39] E. György, A. Pérez del Pino, C. Logofatu, C. Cazan and A. Duta, Journal of the American Ceramic Society 2014, 97, $2718-2724$.

[40] M. Fan, Z.-Q. Feng, C. Zhu, X. Chen, C. Chen, J. Yang and D. Sun, Journal of Materials Science 2016, 51, 10323-10349.

[41] N. A. Kumar, H. Nolan, N. McEvoy, E. Rezvani, R. L. Doyle, M. E. G. Lyons and G. S. Duesberg, Journal of Materials Chemistry A 2013, 1, 4431-4435.

[42] N. K. Stefania Sandoval, Judith Oro-Solé, A. Sundaresan, C. N. R. Rao, Amparo Fuertes, Gerard Tobias, Carbon 2016, 96, 594-602.

[43] F. M. Hassan, V. Chabot, J. Li, B. K. Kim, L. Ricardez-Sandoval and A. Yu, Journal of Materials Chemistry A 2013, 1, $2904-2912$.

[44] S. M. Unni, S. Devulapally, N. Karjule and S. Kurungot, Journal of Materials Chemistry 2012, 22, 23506-23513.

[45] D. Long, W. Li, L. Ling, J. Miyawaki, I. Mochida and S. H. Yoon, Langmuir 2010, 26, 16096-16102.

[46] C. Zhu, S. Guo, Y. Fang and S. Dong, ACS Nano 2010, 4, 2429-2437.

[47] a) X. Teng, M. Yan and H. Bi, Spectrochimica Acta - Part A: Molecular and Biomolecular Spectroscopy 2014, 118, 1020-1024;b) Y. Zhang, L. Wang, D. Lu, X. Shi, C. Wang and X. Duan, Electrochimica Acta 2012, 80, 77-83;c) S. Spanò, G. Isgrò, P. Russo, M. Fragalà and G. Compagnini, Applied Physics A 2014, 117, 19-23 